\title{
Clinical Study \\ Prevention of Peritoneal Metastases from Colon Cancer in High-Risk Patients: Preliminary Results of Surgery plus Prophylactic HIPEC
}

\author{
Paolo Sammartino, ${ }^{1}$ Simone Sibio, ${ }^{1}$ Daniele Biacchi, ${ }^{1}$ Maurizio Cardi, ${ }^{1}$ Fabio Accarpio, ${ }^{1}$ \\ Pietro Mingazzini, ${ }^{2}$ Maria Sofia Rosati, ${ }^{2}$ Tommaso Cornali, ${ }^{1}$ and Angelo Di Giorgio' \\ ${ }^{1}$ Dipartimento di Chirurgia Pietro Valdoni, Azienda Policlinico Umberto I, Università degli Studi di Roma "La Sapienza," \\ 00161 Roma, Italy \\ ${ }^{2}$ Dipartimento di Medicina Sperimentale, Azienda Policlinico Umberto I, Università degli Studi di Roma "La Sapienza," \\ 00161 Roma, Italy
}

Correspondence should be addressed to Paolo Sammartino, paolo.sammartino@uniroma1.it

Received 26 January 2012; Accepted 17 February 2012

Academic Editor: Paul H. Sugarbaker

Copyright (C) 2012 Paolo Sammartino et al. This is an open access article distributed under the Creative Commons Attribution License, which permits unrestricted use, distribution, and reproduction in any medium, provided the original work is properly cited.

The study compared the outcome in patients with advanced colonic cancer at high risk of peritoneal metastases (mucinous or signet-ring cell) without peritoneal or systemic spread, treated with standard colectomy or a more aggressive combined surgical approach. The study included patients with colonic cancer with clinical T3/T4, any N, M0, and mucinous or signet ring cell histology. The 25 patients in the experimental group underwent hemicolectomy, omentectomy, bilateral adnexectomy, hepatic round ligament resection, and appendectomy, followed by HIPEC. The control group comprised 50 patients treated with standard surgical resection during the same period in the same hospital by different surgical teams. Outcome data, morbidity, peritoneal recurrence rate, and overall, and disease-free survival, were compared. Peritoneal recurrence developed in $4 \%$ of patients in the experimental group and $22 \%$ of controls without increasing morbidity $(P<0.05)$. Actuarial overall survival curves disclosed no significant differences, whereas actuarial disease-free survival curves showed a significant difference between groups (36.8 versus 21.9 months, $P<0.01)$. A more aggressive preventive surgical approach combined with HIPEC reduces the incidence of peritoneal recurrence in patients with advanced mucinous colonic cancer and also significantly increases disease-free survival compared with a homogeneous control group treated with a standard surgical approach without increasing morbidity.

\section{Introduction}

Epidemiological data indicate that peritoneal spread from colorectal cancer is an event that involves $10-15 \%$ of patients at the time of primary cancer resection and about $25-50 \%$ of patients with recurrent disease, generally leading to death within weeks or months [1-5]. Several features of primary tumors of colorectal origin appear to be related to a later development of peritoneal spread: mucinous colorectal cancers or signet ring cell carcinomas tend preferentially to metastasize to the peritoneum or ovaries [6-10]. Intraperitoneal metastases may spread by full-thickness bowel wall invasion or may arise iatrogenically during surgery when "in transit" tumor cells or emboli escape from dissected lymph vessels within the bowel lumen or reach the peritoneal cavity through blood spill from the surgical field [11].

Since the 1990s, Paul Sugarbaker's studies on cytoreductive surgery plus perioperative intraperitoneal chemotherapy such as hyperthermic intraperitoneal chemotherapy (HIPEC) have prompted a new treatment option for selected pa-tients with peritoneal metastases from colorectal cancer [12-14]. Three studies, one randomized and two nonrandomized, have shown that this combined procedure provides a better outcome than 5-fluorouracil-based chemotherapy or more modern chemotherapy regimens [15-17].

Despite these encouraging results, even if 5-year survival can reach a value close to $45 \%$ at the expense of a mortality rate ranging from 3 to $5 \%$ and a morbidity rate around $30 \%$ 
in selected cases $[18,19]$, outcome depends on many factors. The first is peritoneal involvement as measured by the peritoneal cancer index (PCI), the second the degree of cytoreduction achieved, and finally the surgical team's level of experience $[20,21]$. Given that better prognostic results can be expected only in patients with a low PCI, in whom complete cytoreduction is possible, and because current imaging techniques cannot detect peritoneal metastases before they becomes clinically evident and symptomatic $[22,23]$, in practice these combined approaches apply only to few patients and rarely offer long-term survival.

In the past, in line with what others now propose for ovarian cancer [24], experimental investigations and clinical trials have used normothermic intraperitoneal chemotherapy as adjuvant treatment in colorectal patients at high risk of recurrence, with inconclusive results [25-27]. In recent years, some have suggested early second-look surgery in the absence of clinical signs of recurrence for colorectal patients at high risk of peritoneal relapse to detect and treat those with carcinomatosis at an initial stage $[28,29]$. Based on preliminary results, two randomized trials will begin in France and the United States to answer the question whether second-look surgery envisaging cytoreductive surgery with HIPEC or HIPEC alone will prolong overall survival and reduce the risk of relapse compared with standard care (observation) in patients with colorectal cancer at high risk for peritoneal spread $[30,31]$.

Prompted by the need to seek new ways of managing colorectal cancer in patients at high risk of recurrence but still without evident signs of peritoneal spread, we decided to concentrate our efforts on a timely strategy envisaging a primary operation aimed at preventing peritoneal metastases. Ample evidence shows that the two major elements influencing peritoneal spread in colorectal cancer are the depth of bowel wall invasion (pT3/4) and histological features of the malignancy (mucinous and signet ring cell carcinomas) [5-11]. Both are characteristics that the surgeon can verify during the primary operation and if necessary use the information to change the strategy to a more aggressive approach combining surgical resection and HIPEC.

We designed this single-center case-control study to analyze outcome in two comparable groups of patients with advanced colonic cancer (pT3/4 with mucinous or signet ring cell cancer) without peritoneal or systemic spread treated with standard colectomy, according to the established guidelines [32] (control group), or by a more aggressive combined approach aimed to prevent peritoneal spread. For this purpose, in the experimental group we extended our standard sur-gical resection to the metastatic sanctuaries of peritoneal diffusion (including the omentum, adnexa, and appendix) and combined these surgical procedures with prophylactic HIPEC. As the primary outcome variables we compared morbidity, the incidence of peritoneal recurrence and overall and disease-free survival in the two groups.

\section{Materials and Methods}

2.1. Experimental Group. The study included patients with colonic cancer or intraperitoneal rectosigmoid cancer (over
$15 \mathrm{~cm}$ from the anal verge) with clinical T3/T4, any N, and M0 stage treated at the Department of Surgery Pietro Valdoni at Sapienza University of Rome from January 2006 to December 2008. To avoid bias from neoadjuvant therapy we excluded patients with extraperitoneal rectal cancer. Selection criteria were age younger than 70 years, cancer with mucinous or signet ring cell components ( $>20 \%$ according to criteria proposed by Ogino et al. [33], performance status 0-2 (WHO) [34]), and adequate renal, hepatic, and bone marrow function. All patients gave specific informed written consent. Exclusion criteria were metastatic disease, other malignances, multiple colorectal cancer, active infections, or severe associated medical conditions. Patients with perforated cancers were considered eligible regardless of histology. At surgical exploration, patients with unrecognized peritoneal seedings were excluded from the study as well as those with hepatic involvement detected at intraoperative ultrasound. None of the patients underwent peritoneal lavage cytology [35]. At operation, after the standard hemicolectomy done according to the established guidelines, intraoperative pathologic evaluation assessed tumor depth $(\mathrm{pT})$ and the histologic features necessary to include the patient in the study. In the selected cases the surgical resection also included complete omentectomy, bilateral adnexectomy in postmenopausal patients, hepatic round ligament resection and appendectomy if not already done. At the end of surgery, HIPEC was delivered with the closed technique with oxaliplatin $460 \mathrm{mg} / \mathrm{m}^{2}$ in $21 / \mathrm{m}^{2}$ of dextrose at a temperature of $43^{\circ} \mathrm{C}$ over 30 minutes at a flow rate of $2 \mathrm{~L} / \mathrm{min}$. Before HIPEC began and during surgery patients received intravenous fluorouracil of $400 \mathrm{mg} / \mathrm{m}^{2}$ and leucovorin of $20 \mathrm{mg} / \mathrm{m}^{2}$ to potentiate oxaliplatin activity. Systemic adjuvant chemotherapy was reserved after discharge to patients with pT4, node positive, and G3 tumors. The study was approved by the hospital institutional review board.

2.2. Control Group. Control subjects were retrospectively selected from patients with colonic cancer treated with standard surgical resection, during the same period in the same hospital but by different surgical teams. The selection process comprised two steps. During the first step surgeons from another surgical team in our hospital selected from their records all patients with colonic cancer treated from January 2006 to December 2008 and who met the eligibility criteria required in our experimental study and had known followup. In particular, we selected patients with T3/T4 mucinous or signet ring cell carcinoma resected for cure (R0) without systemic spread. As in the experimental group, control patients with perforated colon cancer were included regardless of histology. During the second step the principal investigator (P. Sammartino) double-checked the medical records for the potentially eligible patients provided by other surgical teams by recontacting the investigator to ensure that the eligibility criteria had been homogeneously applied. During double-checking the investigator was unaware of the patients' outcome.

2.3. Follow-Up and Statistics. Data for patients in the experimental group were recorded prospectively in a specific 
database. Data for patients the control group were recorded retrospectively. Surgical complications and adverse events were monitored in both groups and graded from 0 to $\mathrm{V}$ in accordance with the National Cancer Institute Common Toxicity Criteria [36]. Follow-up assessments took place every 3 months with clinical evaluation and tumor marker monitoring. A 64-section multidetector computed tomography (MDCT) and magnetic resonance imaging (MRI) with conventional and diffusion-weighted sequences were obtained alternatively every 6 months in the experimental and control groups, according to a protocol developed in collaboration with a dedicated radiological team [37]. The definition of peritoneal recurrence, included imaging findings of locoregional progression as well as peritoneal metastases distant from the resection site. No patient was excluded from the survival analysis. The chi-square test was used for univariate comparison. Survival curves were calculated with the Kaplan-Meier method and compared with the log-rank test. Survival was measured from the date of surgical treatment until death or the last follow-up. $P$ values $<0.05$ were considered to indicate statistical significance.

\section{Results}

Of the 230 patients with colonic cancer treated in our department between January 2006 and December 2008, 25 fulfilled the inclusion criteria and agreed to take part in this experimental investigation. A total of 75 patients were proposed for matching with those in the experimental study and after double checking for eligible criteria 50 were included in the control group. The clinical characteristics for both groups are shown in Table 1. Surgical procedures performed in both groups are reported in Table 2 .

When surgery ended all 25 patients in the experimental group underwent HIPEC. In the study group a mean of 20 lymph nodes per patient were removed (range 15-28) and in the control group a mean of 19 (range 14-31). Locoregional lymph node metastases were found in $34 \%$ of patients in the experimental group and in $28 \%$ of those in the control group. Anatomopathological studies in the experimental group showed that none of the surgical specimens excised according to the protocol contained malignant disease. All the surgical procedures in both groups were R0. The mean length of surgery, blood loss, and postoperative stay were similar in the two groups. Except for 1 patient in the experimental group who had grade 2 pancreatitis related to HIPEC toxicity (that promptly regressed after medical therapy) morbidity rates were similar in the two groups. One patient in the experimental group underwent emergency laparotomy on postoperative day 2 for bleeding. One patient in the control group had a grade III complication (left ureteral leakage) that required endoscopy to place a stent, and 3 patients underwent a second laparotomy to construct an ileostomy for anastomotic leakage (Table 3). A total of 13 patients in the experimental group (52\%) and 23 in the control group (46\%) underwent first-line systemic adjuvant chemotherapy with fluorouracil and oxaliplatin. In relapsed patients second-line chemotherapy included irinotecan or
TABLE 1: Clinical characteristics of the 2 groups.

\begin{tabular}{|c|c|c|c|c|}
\hline & \multirow{2}{*}{\multicolumn{2}{|c|}{$\begin{array}{c}\text { Patients }(25) \\
\text { Mean age } 62(45-70)\end{array}$}} & \multirow{2}{*}{\multicolumn{2}{|c|}{$\begin{array}{c}\text { Controls }(50) \\
\text { Mean age } 63(48-72)\end{array}$}} \\
\hline & & & & \\
\hline & $N$ & $\%$ & $N$ & $\%$ \\
\hline \multicolumn{5}{|l|}{ Sex } \\
\hline Male & 16 & 66 & 31 & 62 \\
\hline Female & 9 & 34 & 19 & 38 \\
\hline \multicolumn{5}{|l|}{ Performance status } \\
\hline 0 & 21 & 84 & 41 & 82 \\
\hline 1 & 4 & 16 & 7 & 14 \\
\hline 2 & - & - & 2 & 4 \\
\hline \multicolumn{5}{|l|}{ Tumor site } \\
\hline Right colon & 9 & 36 & 15 & 30 \\
\hline Transverse colon & 3 & 12 & 6 & 12 \\
\hline Left colon & 13 & 52 & 29 & 58 \\
\hline \multicolumn{5}{|l|}{$\mathrm{Pt}$} \\
\hline pT3 & 19 & 76 & 40 & 80 \\
\hline pT4a & 1 & 4 & 1 & 2 \\
\hline pT4b & 5 & 20 & 9 & 18 \\
\hline \multicolumn{5}{|l|}{ Nodal status } \\
\hline N0 & 16 & 66 & 36 & 72 \\
\hline N1-2 & 9 & 34 & 14 & 28 \\
\hline \multicolumn{5}{|l|}{ Grading } \\
\hline G2 & 17 & 68 & 37 & 64 \\
\hline G3 & 8 & 32 & 13 & 26 \\
\hline \multicolumn{5}{|l|}{ Histology } \\
\hline Mucinous & 23 & 92 & 45 & 90 \\
\hline Signet ring cell & 1 & 4 & 4 & 8 \\
\hline Adc nos & $1^{*}$ & 4 & $1^{*}$ & 2 \\
\hline
\end{tabular}

${ }^{*}$ Perforated patients.

molecular target drugs (cetuximab and bevacizumab or both).

3.1. Follow-Up. After a mean 37.8-month follow-up in the experimental group and 35.1 months in the control group, $24 \%$ in the experimental group and $32 \%$ of the controls had recurrent disease (Table 4).

3.2. Experimental Group. Six patients showed relapse of disease: 5 had hepatic and or pulmonary metastases (mean time of recurrence 13 months) and 1 developed a peritoneal recurrence. This patient underwent a right hemicolectomy with abdominal wall resection for a T4b tumour and experienced a peritoneal recurrence detected at 30 months after operation. Two patients (one with single hepatic metastases and the peritoneal recurrence) underwent a second surgical procedure and are at the moment alive and disease-free at 38 and 39 months. Of the other 4 patients, 1 is currently alive with hepatic and pulmonary metastases and 3 died from progressive disease at a mean of 21 months. 
TABLE 2: Surgical procedures performed in the 2 groups.

\begin{tabular}{lcc}
\hline & Patients & Controls \\
\hline Surgical procedures & & \\
Complete omentectomy & $25^{*}$ & - \\
Hepatic round ligament resection & $25^{*}$ & - \\
Left hemicolectomy & 13 & 29 \\
Appendectomy & $10^{*}$ & - \\
Right hemicolectomy & 9 & 15 \\
Bilateral adnexectomy & $6^{*}$ & $1^{\circ}$ \\
Transverse colon resection & 3 & 6 \\
Cholecystectomy & $2^{\S}$ & $2^{\S}$ \\
Abdominal wall resection & $2^{\circ}$ & $3^{\circ}$ \\
Small bowel resection & $2^{\circ}$ & $3^{\circ}$ \\
Right adnexectomy & $1^{*}$ & $1^{\circ}$ \\
Hysterectomy & $1^{\S}$ & $1^{\circ}$ \\
Total, mean per patient & $99-3.9$ & $61-1.2$ \\
\hline
\end{tabular}

* Procedures performed according to the study protocol.

${ }^{\circ}$ Procedures performed for the tumor direct invasion.

$\S$ Procedures performed for the coexisting benign disease.

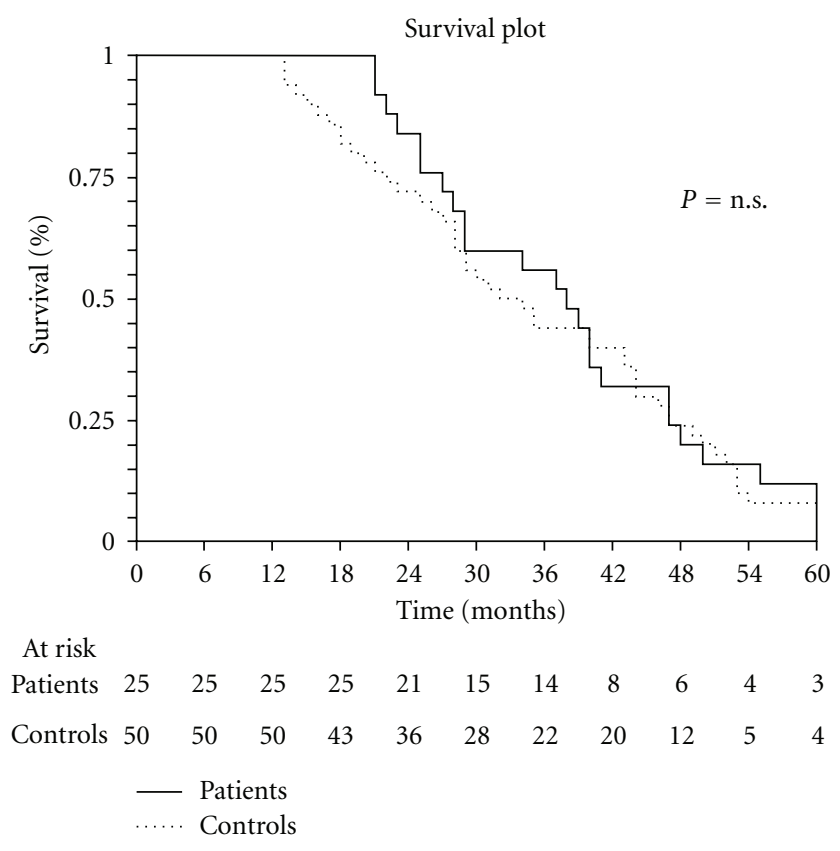

FIGURE 1: Overall survival.

3.3. Control Group. Sixteen patients showed recurrent disease: a peritoneal recurrence was found in 11 (22\%), associated in 4 cases with hepatic and/or pulmonary disease at a mean of 12.7 months after first operation. Initial diagnosis of peritoneal metastases was made after MDCT and MRI findings according to our published protocol [37]. In 9 patients the diagnosis was also confirmed by endoperitoneal ascites cytology or histology during laparoscopy or operation. Five patients showed only a systemic progression of the disease (hepatic in 3, pulmonary in 1 , and both in 1). Three patients underwent a second surgical procedure:

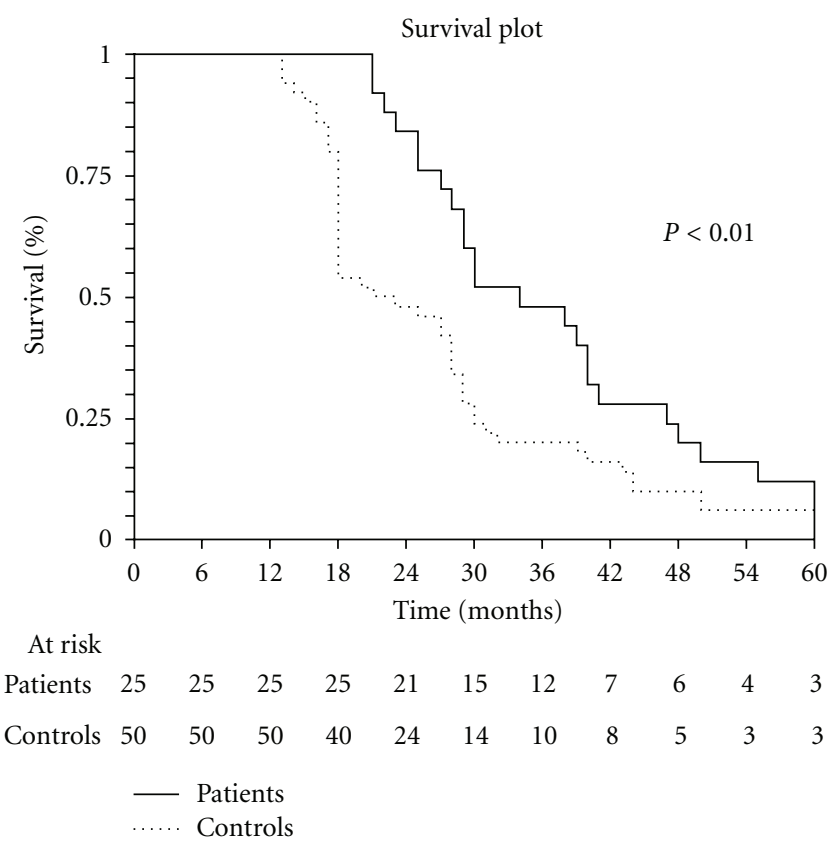

Figure 2: Disease free survival.

1 underwent a wedge resection for a single hepatic metastasis (alive disease-free at 31 months) and 2 cytoreduction and HIPEC for peritoneal metastases (alive with disease at 26 and 24 months). Four patients are currently alive with disease and 9 died from progression at a mean of 15.6 months.

A statistically significant difference in development of peritoneal metastases was observed between the 2 groups $(P<0.05$, Table 4$)$. The actuarial overall survival curves disclose no significant difference between the two groups (Figure 1) whereas the actuarial disease-free survival curves already show a significant difference $(P<0.01)$ between the two groups (36.8 months in the experimental group versus 21.9 months in the control group, Figure 2).

\section{Discussion}

Our preliminary results in this single-center case-control study show that our more aggressive preventive surgical approach combined with HIPEC significantly reduces the incidence of peritoneal recurrence in patients with advanced mucinous colonic cancer and also significantly increases disease-free survival compared with a homogeneous control group treated with a standard surgical approach and does so without increasing morbidity. Although our current data for overall survival seem as yet to show no difference between the survival curves for the experimental group and controls, the significant difference in disease-free survival suggests that overall survival will eventually differ as follow-up progresses.

Our preventive surgical strategy could have improved outcome because it is based on current knowledge on peritoneal fluid dynamics showing that exfoliated tumor cells from full-thickness tumors especially those from mucinous histotypes colonize specific sites [38, 39]. According to 
TABLE 3: Surgical outcome in the 2 groups.

\begin{tabular}{|c|c|c|c|c|}
\hline \multirow{2}{*}{ Morbidity } & \multicolumn{2}{|c|}{ Patients } & \multicolumn{2}{|c|}{ Controls } \\
\hline & $N$ & $\%$ & $N$ & $\%$ \\
\hline Grade I/II & 3 & 12 & 5 & 10 \\
\hline Grade III & - & - & 1 & 2 \\
\hline Grade IV & 1 & 4 & 3 & 6 \\
\hline \multirow[t]{2}{*}{ Hipec tox. grade 2} & $1^{*}$ & 4 & 一 & 一 \\
\hline & \multicolumn{2}{|c|}{ Patients } & \multicolumn{2}{|c|}{ Controls } \\
\hline Mean operation time (min) & \multicolumn{2}{|c|}{$180(120-210)$} & \multicolumn{2}{|c|}{$155(90-220)$} \\
\hline Mean blood loss (mL) & \multicolumn{2}{|c|}{$210(180-290)$} & \multicolumn{2}{|c|}{$230(150-400)$} \\
\hline Postop. stay (days) & \multicolumn{2}{|c|}{$11(8-14)$} & \multicolumn{2}{|c|}{$14(8-21)$} \\
\hline
\end{tabular}

* Pancreatic.

TABLE 4: Site of recurrence.

\begin{tabular}{lccccc}
\hline & \multicolumn{2}{c}{ Patients (25) } & \multicolumn{2}{c}{ Controls (50) } & $P$ \\
\hline Metastases & $N$ & $\%$ & $N$ & $\%$ & \\
Distant & 5 & 20 & 9 & 18 & $\mathrm{~ns}$ \\
Peritoneal & 1 & 4 & $11^{*}$ & 22 & $<0.05$ \\
Total & 6 & 24 & 16 & 32 & $\mathrm{~ns}$ \\
\hline
\end{tabular}

${ }^{*} 4$ patients had also distant metastases.

a series of well-documented events, as in ovarian cancer progression, malignant spread in colonic cancer preferentially targets the omentum, pelvis, and ileocecal regions. Removing these target organs at the first surgical intervention regardless of whether they are macroscopically involved and following these surgical procedures with HIPEC, both aimed to eradicate microscopic residual disease, seems a reasonable strategy to reduce peritoneal spread.

We cannot say whether the reduced peritoneal recurrence and significantly improved disease-free survival in the experimental group depend on the associated surgical procedures (omentectomy, adnexectomy, appendectomy, and round ligament resection) or on HIPEC. Although pathological studies of the anatomic structures preventively removed in our patients disclosed no evidence of malignant disease, we can reasonably presume that removing these structures and delivering HIPEC both contributed to preventing microscopic peritoneal diffusion $[40,41]$.

Our proposal to address the problem from a new angle, namely, preventing colorectal peritoneal spread, seems to offer a promising alternative to those who recommend an early second look in high-risk patients $[28,31]$. In our preventive study we defined high-risk patients with advanced colonic cancer as those with pT3/4 mucinous or signet ring cell cancer without peritoneal or systemic spread whereas those proposing second-look surgery enrolled a varied population including patients who at the primary intervention already had limited peritoneal carcinomatosis or ovarian metastases [28, 31]. These nonhomogeneous populations will make it difficult to interpret outcomes in the two ongoing randomized trials investigating second-look surgery $[30,31]$. A major concern is whether randomizing patients to secondlook surgery or observation is ethically justifiable given that in a preliminary report Elias et al. at second-look found that more than $50 \%$ of high-risk patients had peritoneal carcinomatosis that clinical and imaging examination left unrecognized [28]. Lastly, another problem related to second-look surgery is that a whole class of patients (those termed at high risk) must be referred to highly specialized tertiary centers (peritoneal surface malignancy treatment centers) so that peritoneal carcinomatosis if found can be properly treated. As cancer surgeons well know, the medical community still regards integrated treatments for peritoneal carcinomatosis with skepticism. And we all know how difficult it is to persuade patients (and their oncologist) to undergo a second intervention that may be lengthy and not without risks in the absence of specific symptoms and documentable clinical evidence. From the viewpoint of feasibility and costs we therefore consider it more appropriate to concentrate our efforts on and invest our resources in preventing peritoneal carcinomatosis right from the primary operation. If our innovative preventive strategy proves therapeutically worthwhile then it could be done in a larger number of surgical centers, would involve a larger number of patients, and might finally change the therapeutic options available to patients with advanced colorectal cancer at risk for peritoneal carcinomatosis [29].

Some might criticize our preventive proposal stating that in patients with advanced colonic cancer with no documented signs of carcinomatosis our aggressive approach could be considered overtreatment. This criticism notwithstanding, our early aggressive approach receives strong support because without increasing morbidity rates it lowers the incidence of peritoneal carcinomatosis and offers better disease-free survival than in a homogeneous sample of patients who received standard surgical treatment. Our preventive approach also accords with Sugarbaker, who recommended after second-look surgery negative for carcinomatosis a procedure analogous to the one we describe here (omentectomy, adnexectomy, and HIPEC) [29]. Hence, in high-risk patients why not use this approach right from the primary surgical intervention. The true therapeutic value of our preventive surgical approach for patients with advanced mucinous colonic cancer awaits confirmation in future randomized multicenter studies. 


\section{Disclosure}

The paper was presented at the Sixth International Symposium on Regional Cancer Therapies Incline Village, Nevada on February 19-21, 2011.

\section{References}

[1] D. Z. J. Chu, N. P. Lang, C. Thompson, P. K. Osteen, and K. C. Westbrook, "Peritoneal carcinomatosis in nongynecologic malignancy. A prospective study of prognostic factors," Cancer, vol. 63, no. 2, pp. 364-367, 1989.

[2] B. Sadeghi, C. Arvieux, O. Glehen et al., "Peritoneal carcinomatosis from non-gynecologic malignancies: results of the EVOCAPE 1 multicentric prospective study," Cancer, vol. 88, no. 2, pp. 358-363, 2000.

[3] D. G. Jayne, S. Fook, C. Loi, and F. Seow-Choen, "Peritoneal carcinomatosis from colorectal cancer," British Journal of Surgery, vol. 89, no. 12, pp. 1545-1550, 2002.

[4] A. Gómez Portilla, I. Cendoya, I. López De Tejada et al., "Peritoneal carcinomatosis of colorectal origin. Current treatment. Review and update," Revista Espanola de Enfermedades Digestivas, vol. 97, no. 10, pp. 716-737, 2005.

[5] V. E. Lemmens, Y. L. Klaver, V. J. Verwaal, H. J. Rutten, J. W.W. Coebergh, and I. H. De Hingh, "Predictors and survival of synchronous peritoneal carcinomatosis of colorectal origin: a population-based study," International Journal of Cancer, vol. 128, no. 11, pp. 2717-2725, 2011.

[6] T. Nozoe, H. Anai, S. Nasu et al., "Clinicopathological characteristics of mucinous carcinoma of the colon and rectum," Journal of Surgical Oncology, vol. 75, no. 2, pp. 103-107, 2000.

[7] Y. Kanemitsu, T. Kato, T. Hirai et al., "Survival after curative resection for mucinous adenocarcinoma of the colorectum," Diseases of the Colon and Rectum, vol. 46, no. 2, pp. 160-167, 2003.

[8] R. Pande, A. Sunga, C. LeVea et al., "Significance of signet-ring cells in patients with colorectal cancer," Diseases of the Colon and Rectum, vol. 51, no. 1, pp. 50-55, 2008.

[9] V. Catalano, F. Loupakis, F. Graziano et al., "Mucinous histology predicts for poor response rate and overall survival of patients with colorectal cancer and treated with first-line oxaliplatin- and/or irinotecan-based chemotherapy," British Journal of Cancer, vol. 100, no. 6, pp. 881-887, 2009.

[10] W. Song, S. J. Wu, Y. L. He et al., "Clinicopathologic features and survival of patients with colorectal mucinous, signetring cell or non-mucinous adenocarcinoma: experience at an institution in southern China," Chinese Medical Journal, vol. 122, no. 13, pp. 1486-1491, 2009.

[11] M. J. Koppe, O. C. Boerman, W. J. G. Oyen, and R. P. Bleichrodt, "Peritoneal carcinomatosis of colorectal origin: incidence and current treatment strategies," Annals of Surgery, vol. 243, no. 2, pp. 212-222, 2006.

[12] P. H. Sugarbaker, "Peritonectomy procedures," Annals of Surgery, vol. 221, no. 1, pp. 29-42, 1995.

[13] P. H. Sugarbaker, Peritoneal Carcinomatosis: Principles of Management, Kluwer Academic, Boston, Mass, USA, 1996.

[14] J. Esquivel, R. Sticca, P. Sugarbaker et al., "Cytoreductive surgery and hyperthermic intraperitoneal chemotherapy in the management of peritoneal surface malignancies of colonic origin: a consensus statement," Annals of Surgical Oncology, vol. 14, no. 1, pp. 128-133, 2007.

[15] V. J. Verwaal, S. Van Ruth, E. De Bree et al., "Randomized trial of cytoreduction and hyperthermic intraperitoneal chemotherapy versus systemic chemotherapy and palliative surgery in patients with peritoneal carcinomatosis of colorectal cancer," Journal of Clinical Oncology, vol. 21, no. 20, pp. 3737-3743, 2003.

[16] D. Elias, J. H. Lefevre, J. Chevalier et al., "Complete cytoreductive surgery plus intraperitoneal chemohyperthermia with oxaliplatin for peritoneal carcinomatosis of colorectal origin," Journal of Clinical Oncology, vol. 27, no. 5, pp. 681-685, 2009.

[17] J. Franko, Z. Ibrahim, N. J. Gusani, M. P. Holtzman, D. L. Bartlett, and H. J. Zeh, "Cytoreductive surgery and hyperthermic intraperitoneal chemoperfusion versus systemic chemotherapy alone for colorectal peritoneal carcinomatosis," Cancer, vol. 116, no. 16, pp. 3756-3762, 2010.

[18] V. J. Verwaal, S. Van Ruth, A. Witkamp, H. Boot, G. Van Slooten, and F. A. N. Zoetmulder, "Long-term survival of peritoneal carcinomatosis of colorectal origin," Annals of Surgical Oncology, vol. 12, no. 1, pp. 65-71, 2005.

[19] D. Elias, B. Raynard, F. Farkhondeh et al., "Peritoneal carcinomatosis of colorectal origin: long-term results of intraperitoneal chemohyperthermia with oxaliplatin following complete cytoreductive surgery," Gastroenterologie Clinique et Biologique, vol. 30, no. 10, pp. 1200-1204, 2006.

[20] D. Elias, F. Gilly, F. Boutitie et al., "Peritoneal colorectal carcinomatosis treated with surgery and perioperative intraperitoneal chemotherapy: retrospective analysis of 523 patients from a multicentric french study," Journal of Clinical Oncology, vol. 28, no. 1, pp. 63-68, 2010.

[21] C. Cao, T. D. Yan, D. Black, and D. L. Morris, "A systematic review and meta-analysis of cytoreductive surgery with perioperative intraperitoneal chemotherapy for peritoneal carcinomatosis of colorectal origin," Annals of Surgical Oncology, vol. 16, no. 8, pp. 2152-2165, 2009.

[22] C. Dromain, S. Leboulleux, A. Auperin et al., "Staging of peritoneal carcinomatosis: enhanced CT vs. PET/CT," Abdominal Imaging, vol. 33, no. 1, pp. 87-93, 2008.

[23] J. L. Koh, T. D. Yan, D. Glenn, and D. L. Morris, "Evaluation of preoperative computed tomography in estimating peritoneal cancer index in colorectal peritoneal carcinomatosis," Annals of Surgical Oncology, vol. 16, no. 2, pp. 327-333, 2009.

[24] L. Elit, T. K. Oliver, A. Covens et al., "Intraperitoneal chemotherapy in the first-line treatment of women with stage III epithelial ovarian cancer: a systematic review with metaanalyses," Cancer, vol. 109, no. 4, pp. 692-702, 2007.

[25] W. Scheithauer, G. V. Kornek, A. Marczell et al., "Combined intravenous and intraperitoneal chemotherapy with fluorouracil + leucovorin vs fluououracil + levamisole for adjuvant therapy of resected colon carcinoma," British Journal of Cancer, vol. 77, no. 8, pp. 1349-1354, 1998.

[26] J. C. Vaillant, B. Nordlinger, S. Deuffic et al., "Adjuvant intraperitoneal 5-fluorouracil in high-risk colon cancer: a multicenter phase III trial," Annals of Surgery, vol. 231, no. 4, pp. 449-456, 2000.

[27] B. Nordlinger, P. Rougier, J. P. Arnaud et al., "Adjuvant regional chemotherapy and systemic chemotherapy versus systemic chemotherapy alone in patients with stage II-III colorectal cancer: a multicentre randomised controlled phase III trial," Lancet Oncology, vol. 6, no. 7, pp. 459-468, 2005.

[28] D. Elias, D. Goéré, D. Di Pietrantonio et al., "Results of systematic second-look surgery in patients at high risk of developing colorectal peritoneal carcinomatosis," Annals of Surgery, vol. 247, no. 3, pp. 445-450, 2008.

[29] P. H. Sugarbaker, "Comprehensive management of disseminated colorectal cancer," Annals of Surgical Oncology, vol. 15, no. 12, pp. 3327-3330, 2008. 
[30] L. Maggiori and D. Elias, "Curative treatment of colorectal peritoneal carcinomatosis: current status and future trends," European Journal of Surgical Oncology, vol. 36, no. 7, pp. 599603, 2010.

[31] R. T. Ripley, J. L. Davis, C. D. Kemp, S. M. Steinberg, M. A. Toomey, and I. Avital, "Prospective randomized trial evaluating mandatory second look surgery with HIPEC and CRS vs. standard of care in patients at high risk of developing colorectal peritoneal metastases," Trials, vol. 11, article no. 62, 2010.

[32] H. Nelson, N. Petrelli, A. Carlin et al., "Guidelines 2000 for colon and rectal cancer surgery," Journal of the National Cancer Institute, vol. 93, no. 8, pp. 583-596, 2001.

[33] S. Ogino, M. Brahmandam, M. Cantor et al., "Distinct molecular features of colorectal carcinoma with signet ring cell component and colorectal carcinoma with mucinous component," Modern Pathology, vol. 19, no. 1, pp. 59-68, 2006.

[34] World Health Organization, WHO Handbook for Reporting Results of Cancer Traetment, WHO, Geneva, Switzerland, 1979.

[35] J. H. Lefevre and D. M. Elias, "Cytoreductive surgery plus intraperitoneal chemohyperthermia in patients with colorectal cancer at high risk for local-regional recurrence," Cancer Journal, vol. 15, no. 3, pp. 200-203, 2009.

[36] A. Saxena, T. D. Yan, and D. L. Morris, "A critical evaluation of risk factors for complications after cytoreductive surgery and perioperative intraperitoneal chemotherapy for colorectal peritoneal carcinomatosis," World Journal of Surgery, vol. 34, no. 1, pp. 70-78, 2010.

[37] F. Iafrate, M. Ciolina, P. Sammartino et al., "Peritoneal carcinomatosis: imaging with 64-MDCTand 3T MRI with diffusion-weighted imaging," Abdominal Imaging. In press.

[38] C. P. Carmignani, T. A. Sugarbaker, C. M. Bromley, and P. H. Sugarbaker, "Intraperitoneal cancer dissemination: mechanisms of the patterns of spread," Cancer and Metastasis Reviews, vol. 22, no. 4, pp. 465-472, 2003.

[39] P. H. Sugarbaker, “Mucinous colorectal carcinoma," Journal of Surgical Oncology, vol. 77, no. 4, pp. 282-283, 2001.

[40] P. H. Sugarbaker, "It's what the surgeon doesn't see that kills the patient," Journal of Nippon Medical School, vol. 67, no. 1, pp. 5-8, 2000.

[41] S. A. Gerber, V. Y. Rybalko, C. E. Bigelow et al., "Preferential attachment of peritoneal tumor metastases to omental immune aggregates and possible role of a unique vascular microenvironment in metastatic survival and growth," American Journal of Pathology, vol. 169, no. 5, pp. 1739-1752, 2006. 


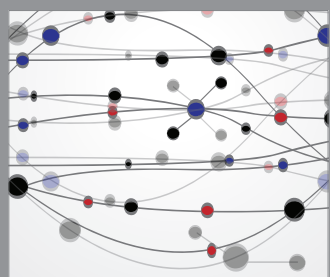

The Scientific World Journal
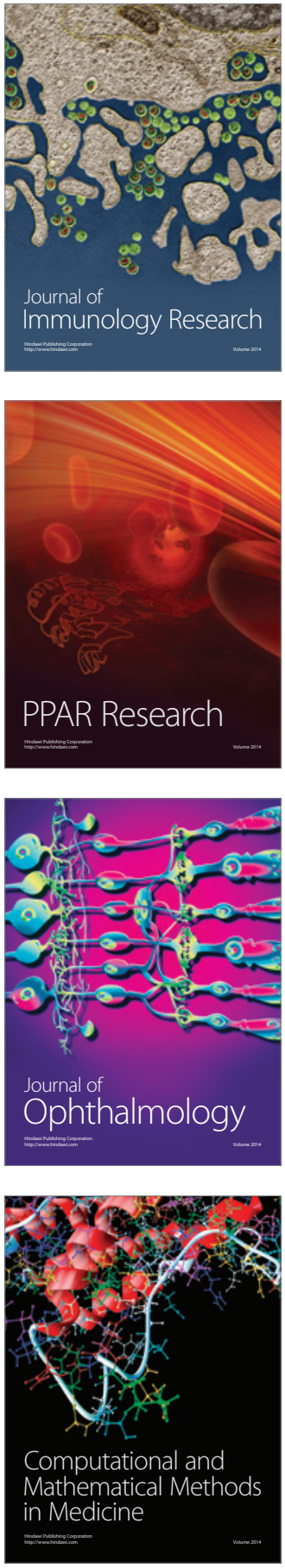

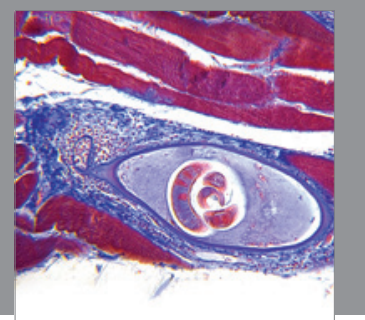

Gastroenterology

Research and Practice
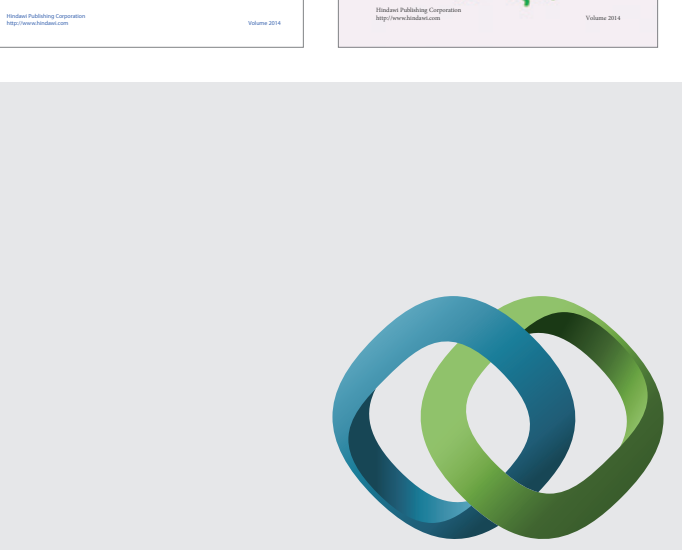

\section{Hindawi}

Submit your manuscripts at

http://www.hindawi.com
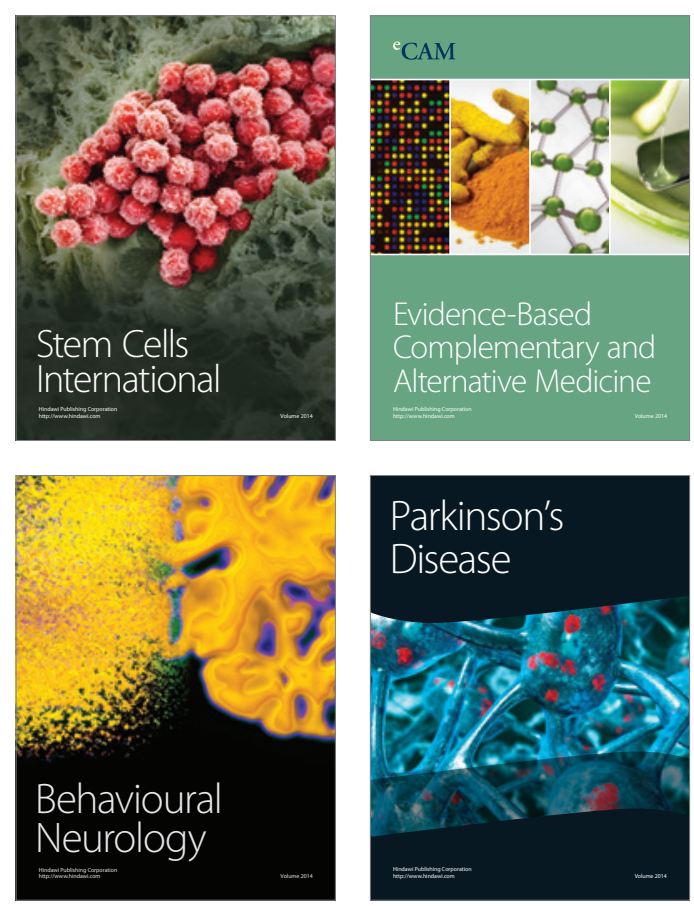

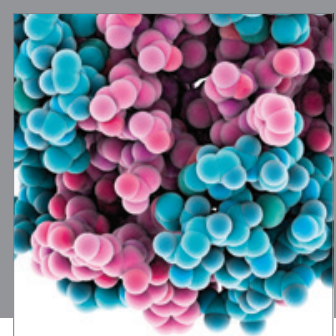

Journal of
Diabetes Research

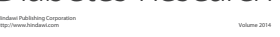

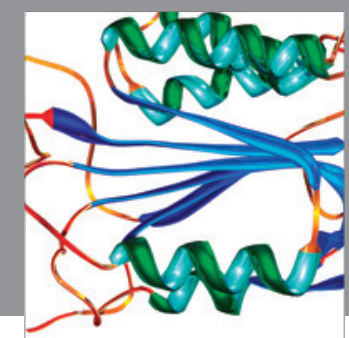

Disease Markers
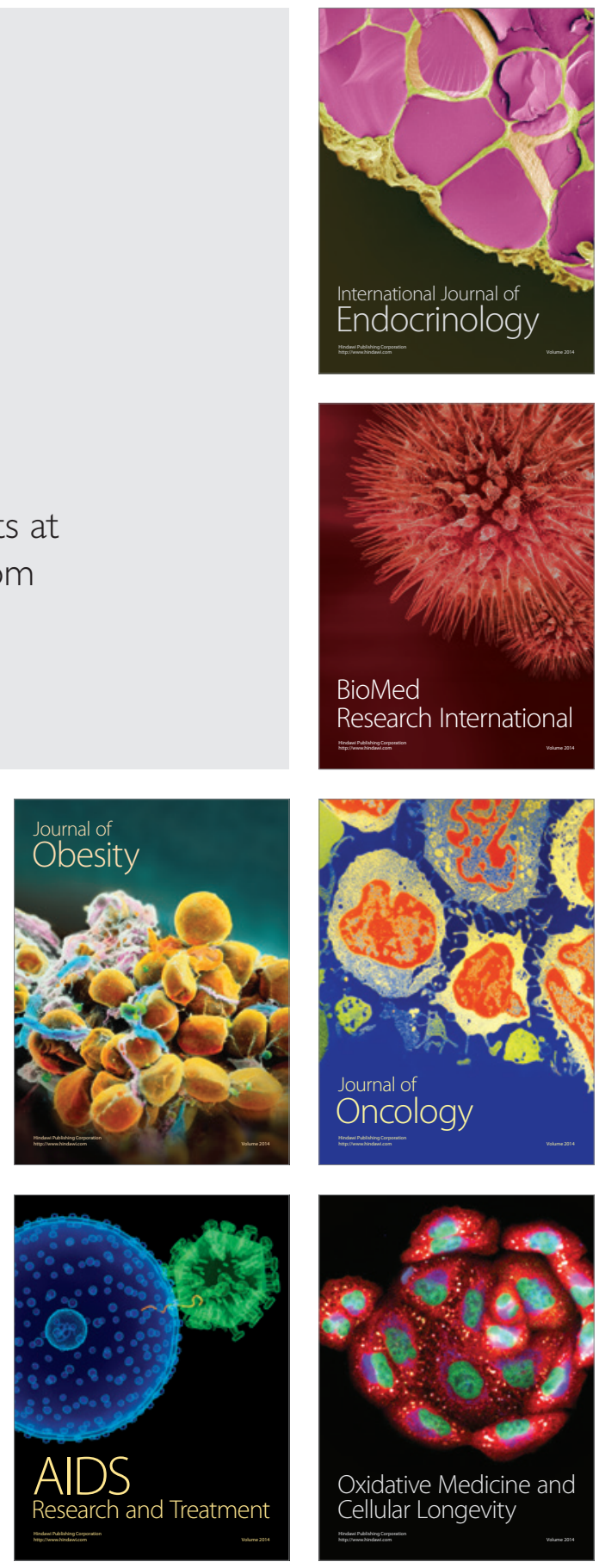